\title{
Development and research of centrifugal mixer for baby food
}

\author{
Dmitrii Sukhorukov ${ }^{*}$, Dmitrii Borodulin ${ }^{1}$, and Diana Cherkashina ${ }^{1}$ \\ ${ }^{1}$ Institute of Engineering Technologies, Kemerovo State University, 6, Krasnaya St., Kemerovo, \\ 650000, Russia
}

\begin{abstract}
The study was carried out on two mixers of different designs: basic and developed. To evaluate and analyze the finished mixture, the method of high performance liquid chromatography was used. Taurine was used as a key component. The paper describes how the rational parameters of the mixer were selected, according to which recipes the mixture was prepared, and also how the final assessment of the effectiveness of the mixing equipment was carried out. The mixtures were prepared on the equipment according to three recipes. According to the first recipe, 81 experiments were carried out, with various parameters of the mixer operation, which helped to determine their rational value. For two different mixers, they turned out to be identical, so further mixing took place with the same parameters. Based on the data obtained, graphs of dependences were built, which clearly demonstrated the difference in the obtained values of the coefficient of heterogeneity. And they made it possible to judge the influence of each individual parameter. The developed mixer design showed a lower coefficient of heterogeneity in comparison with the base one. It did not exceed $10 \%$, unlike the latter.
\end{abstract}

\section{Introduction}

The definition of a balanced diet for baby food should be consistent with the physiological needs and individual characteristics of the child. A wide variety of infant formula exists on the market today. Adapted demineralized whey mixes are in the highest demand. They are close in composition to breast milk. The composition of the mixture should contain a vitamin and mineral complex and various bioactive additives. This is necessary in order to ensure the correct development of the child and to replenish all the necessary substances in the body. The quality of the mixture strongly depends not only on the components that make up its composition, but also on the very process of its preparation. The main problem lies in the uniform distribution of various additives introduced in small amounts throughout the mixture [1].

Mixers play a special role in the technological process of mixing. Their main task is to ensure the uniform distribution of the components in the mixture. Today, centrifugal mixers are most widely used, since they have a number of significant advantages. Such apparatuses provide good mixing quality of light powdery bulk materials; have high performance; have

* Corresponding author: pioner dias@mail.ru 
the ability to combine several processes in one device; characterized by a relatively short mixing cycle and low energy consumption. The use of continuous mixers is most appropriate for the production of mixtures on an industrial scale, since they can be automated and easier to maintain [2]. This consists in the fact that the loading of the components, their mixing and the delivery of the finished mixture are carried out simultaneously and continuously. In order to ensure the optimal composition of infant formula, it is necessary to improve the technological line for its production. This will improve the quality of the product and solve the problem of a complete, balanced nutrition for the child.

The purpose of this work is to develop mixing equipment, which is part of the technological line for the production of infant formula.

In accordance with the purpose of the following tasks:

- investigation of existing types of baby food;

- investigation of equipment for the production of dry infant formula to determine their main advantages and disadvantages, development trends;

- development of a new continuous centrifugal mixer for the production of dry infant formula;

- research of the developed centrifugal mixer to identify the main technological parameters of the equipment.

\section{Materials and methods}

Any production is strictly regulated by federal laws and state standards. There are a number of federal laws, such as "On Protection of Consumer Rights", "On Standardization", "On Certification of Products and Services", "On the Sanitary and Epidemiological Well-Being of the Population", "On the Quality and Safety of Food Products", which allow regulating quality issues and product safety at the legislative level.

They are especially strict about this issue in the production of such significant products as baby food. Requirements for its production and sale are much higher than for adult food products, and the resulting relationships are regulated by many regulatory documents.

Increased hygiene requirements are imposed on the quality of products for newborns and young children, since they have insufficiently developed immune and enzymatic systems.

When compiling a multicomponent mixture for children's products, each dairy component is checked according to a set of physical and chemical indicators: mass fractions of fat and protein, acidity, density, heat resistance, etc. Also introduced components of nondairy origin are periodically controlled: flour, sugar, corn oil, minerals, vitamins.

Serious microbiological and sanitary-hygienic control at all stages of production is required for baby food that include some microbiological indicators.

Control is carried out at least once every 10 days during the technological process. Such control is carried out daily until the causes of bacterial contamination are identified and eliminated in case of obtaining non-standard microbiological data on the finished product.

The quality of equipment washing is mandatory, both at the site of unpasteurized components and at the line of pasteurized milk and mixtures of fermented milk products and starter cultures, which is carried out daily. The quality of hand washing of workers is monitored at least three times a month. The workers' hands are examined daily for cuts, pustular diseases; sanitary clothing is checked two to three times a week. Periodically, in accordance with the sanitary and hygienic state of production, the quality of drinking water and indoor air are examined [3].

The use of biologically active additives based on medicinal and wild herbs when feeding young children is not allowed. Exceptions are dill, fennel and chamomile. Baby 
food products for young children should not contain: flavorings, dyes, stabilizers, preservatives, sugar substitutes, food additives, except for those permitted for the production of baby food [4-6]. There are also a number of federal laws that must be taken into account when manufacturing products for children. These include the previously mentioned Federal Law № 29 - "On the Quality and Safety of Food Products", Federal Law № 52 - "On the Sanitary and Epidemiological Welfare of the Population", as well as Federal Law № 184 - "On Technical Regulation", Federal Law № 163 - "On Amendments to the Federal Law" Technical Regulations for Milk and Dairy Products ", Federal Law № 178 -" Technical Regulations for Fat and Oil Products ". Within the framework of the Customs Union, Technical Regulations were developed. They are as close as possible to the requirements of European and international standards, which are currently the main regulatory documents in the development and assessment of product quality. All this huge list of regulatory documents allows you to control the quality of products and ensure the protection of the life and health of children. They establish mandatory requirements for the production and sale of food products intended for baby food.

Centrifugal mixers are used for mixing bulk materials in baby food production. One of them is shown in Fig. 1.

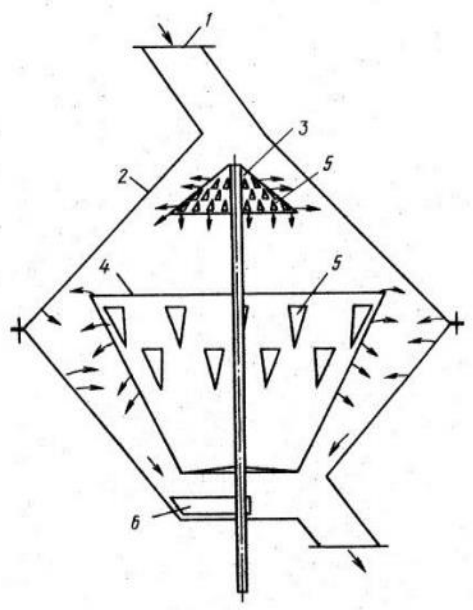

Fig. 1. Basic mixer design: 1 - inlet pipe, 2 - cover, 3 - turbulator, 4 - rotor, 5 - holes, 6 - blade.

This design was used by us as a base for research and further comparative analysis. The mixer works as follows. Bulk materials through the inlet pipe (1) go to the rotating turbulator (3). Under the influence of centrifugal force, material is dropped onto the walls of the housing cover (2). The discharge of material particles occurs from different heights and different accelerations due to the conical shape of the turbulator [7].

Holes (5), located on the surface of the turbulator, additionally divide the mixed material into smallest flows, which, under the action of their own weight and a vortex air flow, move to the bottom of the mixer to the rotor (4). Under the action of centrifugal forces, the mass rises along the cone and, having reached the perforation (5), is thrown out from different heights and at different speeds. Due to this, the material is divided into many streams, which collide with each other in the annular space between the body and the cone (4) and mix intensively. Under the action of their own weight, the particles fall to the bottom of the mixer, from where they are removed by the blade (6).

The mixing process has its own characteristics. It consists of several stages: convective mixing, diffusion mixing and segregation. All three stages occur simultaneously, but if the 
first two help to ensure an even distribution of components in the mixture, then the latter prevents this. At the very beginning of the process, convective mixing prevails at the level of microvolumes. At this stage, the nature of the movement of the working parts of the mixer and, accordingly, the mixture, is of primary importance. After the distribution of the components throughout the volume of the mixer, diffusion mixing, which occurs at the level of microvolumes, is of primary importance [8]. Further, along with diffusion mixing, the segregation of particles begins to significantly affect the mixing process. At some point in time, these processes balance each other, and further mixing is meaningless. The principle of operation of a centrifugal mixer is the interaction of two main forces: centrifugal and friction. The incoming material begins to move along annular paths, drawing neighboring particles into rotation. As a result, vortex flows are formed in the mixer, which ensure constant circulation of substances. The particles collide with each other, moving along different circular paths [9]. Considering the intensification of the process, one should take into account such factors as:

- profile of working bodies,

- frequency of their rotation,

- presence of dead zones in the mixer,

- filling factor of the mixer, etc.

The continuity of the process necessitates not only compliance with the specified concentrations of all components, but also a decrease in their variation at the outlet from the apparatus. The unevenness of the incoming flows can be caused by errors of the dosing device or depend on the structure of materials, it can be of a random or regular order. In any of these cases, the mixer must have good smoothing ability. The purpose of the invention is to intensify the mixing process of bulk materials with a ratio of initial components of 1: 100 and higher, as well as to create an external recirculation of the mixture in the mixer, which will improve the mixing quality of bulk materials. To achieve this goal, it is proposed to make the rotor in the form of a profile cone, which will create a reverse recirculation loop, i.e. will ensure the return of part of the material to the turbulator. Fig. 2 shows a general view of the new design of a continuous centrifugal mixer.

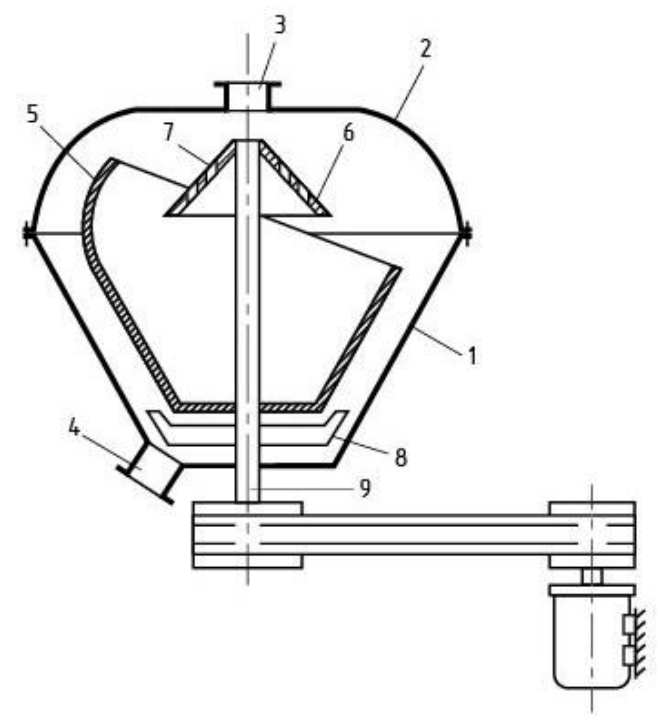

Fig. 2. The developed design of the mixer: 1 - body, 2 - cover, 3 - inlet pipe, 4 - outlet pipe, 5 - rotor, 6 - plate, 7 - holes, 8 - blade, 9 - shaft. 
The mixer works as follows. The material is fed into the mixer through the inlet pipe (3). The rotating plate (6) creates a vortex air flow and, due to its shape and the presence of holes (7), separates the incoming flow. Part of the material is dumped onto the walls of the body, part is poured down the cone, and part passes through the holes. Then the material, under the action of gravity, enters the rotor bowl (5), where, under the action of inertial forces, it begins to rise up along the inner surface. Due to the shape of the rotor, part of the flow returns to the plate (6), and the other part, reaching the smaller edge of the bowl, is dropped onto the surface of the body. The rise of particles along the larger edge of the rotor is ensured by the fact that the profile angle is less than the ascent angle of the particle trajectory along the generatrix of the rotor bowl. As a result, a reverse recirculation loop is formed, and part of the material returns to the surface of the upper cone. Using the blades (8), the finished material is discharged from the mixer through the outlet pipe (4).

Taurine (2-aminoethanesulfonic acid) is the key component of the mixture under study. Taurine is a free-form sulfonic acid. It is an essential component of breast milk, the content of which reaches about 4-5 $\mathrm{mg}$ per $100 \mathrm{ml}$. In the body, the required amount of taurine begins to be produced only when the child reaches about one year old. This valuable substance is involved in the functions:

- construction of the retina and brain;

- maturation and differentiation of tissues of the nervous system;

- increasing the body's immune forces;

- manifestation of antioxidant properties;

- assimilation of minerals (magnesium, sodium, calcium) and fat-soluble vitamins;

- digestion and assimilation of fats;

- elimination of irritability and anxiety.

Taurine is responsible for the active growth and rapid development of the child. The need for its presence in baby food has been scientifically proven. Without it, the mixture will be less complete. The technical regulations for adapted dry milk mixtures set the content of tauric acid not more than $8 \mathrm{mg}$ per $100 \mathrm{ml}$. of liquid product. The total amount of the component in baby food varies from $3,2 \mathrm{mg}$ to $6 \mathrm{mg}$ per $100 \mathrm{ml}$. High performance liquid chromatography method (HPLC) was used to determine the concentration of taurine in infant formula. Currently, this method is widely used to separate and determine the quantitative composition of multicomponent mixtures. The principle of operation of the chromatograph is based on the separation of substances in a chromatographic column. It is a tube filled with a sorbent (stationary phase) through which a solvent (mobile phase) flows continuously. If a substance is introduced into the solvent, then when moving along the column, the molecules of the substance will spread over the sorbent and be adsorbed on its surface. In this case, the time of their adsorption will be determined by the forces of intermolecular interaction. The greater these forces, the slower the molecules of the substance move down the column. At the exit from the column, the concentration of the substance is recorded using a special detector. Based on these data, a chromatogram is constructed, which shows the dependence of the concentration on the retention time of the substance in the column. The detector does not measure the concentration itself, but a value functionally related to the concentration[10].

The liquid chromatograph device includes: a pump that feeds the mobile phase through the column; dispenser for accurate sample injection; separating column; a detector for obtaining an analytical signal; information processing system[11]. Taurine was determined on an Agilent 1200 liquid chromatograph with a spectrophotometric detection system. To facilitate the final analysis of taurine, its derivatization is first carried out. It is carried out using dinitrofluorobenzene in the presence of dimethyl sulfoxide. For laboratory analysis of the finished mixture, 60 samples were taken, each weighing $15 \mathrm{~g}$. Before starting the study, the mixture is restored (in accordance with the instructions) by mixing the dry base with 
warm water (temperature $45^{\circ} \mathrm{C}$ ) and cooled to room temperature. Get $100 \mathrm{ml}$. of the finished mixture.

Measure $3 \mathrm{~cm}^{3}$ of the resulting solution into a $15 \mathrm{~cm}^{3}$ test tube, add $3 \mathrm{~cm}^{3}$ of $3 \%$ sulfosalicylic acid, mix and incubate at room temperature for 20 minutes. Then the tube is placed in a centrifuge for 10 minutes. Pipette off the aqueous layer and pass through a filter. A filtered sample with a volume of $1,0 \mathrm{~cm}^{3}$ is placed in a $10 \mathrm{~cm}^{3}$ measuring tube with a thin

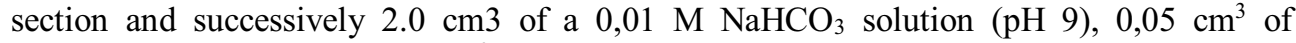
dinitrofluorobenzene and $0,5 \mathrm{~cm}^{3}$ of dimethyl sulfoxide are added, sealed with a stopper and shaken ... Then for 10 minutes incubated in a water bath at $40^{\circ} \mathrm{C}$. Then it is cooled and the total volume of the solution is adjusted to $10 \mathrm{~cm}^{3}$ with phosphate buffer $(\mathrm{pH} \mathrm{7,0)}$. Prepare a standard solution of N1 taurine of known concentration. For this, $50 \mathrm{mg}$ of taurine is placed in a $100 \mathrm{~cm}^{3}$ test tube and made up to the mark with distilled water. Further, by diluting the N1 solution, solutions of the required concentration are obtained. Peak areas are recorded and plotted. The taurine peak is identified by determining the absorption spectrum of the analyte at a wavelength of $360 \mathrm{~nm}$. The processing of chromatograms and the calculation of quantitative characteristics are carried out based on the dependence graph:

$$
C_{\mathrm{x}}=\frac{S_{\mathrm{x}}}{k}
$$

$\mathrm{C}_{\mathrm{x}}$ - mass concentration of taurine in the analyzed sample, $\mathrm{mg} / \mathrm{cm}^{3}$;

$\mathrm{k}$ - calibration factor, $\mathrm{mAU} \cdot \mathrm{min} \cdot \mathrm{cm}^{3} / \mathrm{mg}$;

$\mathrm{S}_{\mathrm{x}}-$ analyte peak area, $\mathrm{mAU} \cdot \mathrm{min}$.

Or using a standard solution:

$$
C_{\mathrm{x}}=\frac{C_{\mathrm{cT}} \cdot S_{\mathrm{x}}}{S_{\mathrm{cT}}},
$$

$\mathrm{C}_{\mathrm{cT}}$ - mass concentration of taurine in standard solution, $\mathrm{mg} / \mathrm{cm}^{3}$;

$\mathrm{S}_{\mathrm{cт}}$ - peak area of a substance in a standard solution, $\mathrm{mAU} \cdot \mathrm{min}$.

The final assessment of the quality of the mixing process was carried out using the coefficient of heterogeneity of the mixture $(\mathrm{Vc})$, It allows you to determine the uniformity of distribution of one of the components in the mixture. This coefficient is calculated by the formula:

$$
\mathrm{Vc}=100 \mathrm{C} \cdot \mathrm{i}=1 \mathrm{nC}-\mathrm{C} 2 \mathrm{n}-1 \mathrm{~V}_{\mathrm{c}}=\frac{100}{\overline{\mathrm{C}}} \cdot \sqrt{\frac{\sum_{i=1}^{\mathrm{n}}\left(\mathrm{C}_{i}-\overline{\mathrm{C}}\right)^{2}}{\mathrm{n}-1}}, \%
$$

$\mathrm{C}_{i}$ - concentration of the $\mathrm{i}$-th sample of the mixture,

$\overline{\mathrm{C}}$ - concentration of a component with its ideal distribution,

$\mathrm{n}$ - number of samples.

The main criterion for the quality indicator of the mixture is the uniform distribution of all its components. Different granulometric composition and other physicochemical properties of the components can create additional difficulties in achieving this result. In order to solve this problem, it is necessary not only to select equipment, but also to properly configure its operation. In order to assess the quality of mixers to be the most effective, three types of mixtures with different content of components were prepared [12-15]. Their composition is presented in table 1. 
Table 1. Formulation of the mixture per $1000 \mathrm{~g}$. of product.

\begin{tabular}{|c|c|c|c|}
\hline Ingredient & Mixture 1 & Mixture 2 & Mixture 3 \\
\hline Dry milk base, g & 650 & 640,5 & 628,6 \\
\hline Diet flour, g & 124,45 & 125,57 & 140 \\
\hline Powdered sugar, g & 210 & 224,7 & 220 \\
\hline Taurine, mg & 390 & 394 & 450 \\
\hline L - Carnitine, mg & 110 & 80 & 110 \\
\hline L - Tryptophan, mg & 1600 & - & - \\
\hline C, mg & 670 & 870 & 680 \\
\hline B1, mg & 3,72 & 5,8 & 3,79 \\
\hline B2, mg & 8,81 & 10,6 & 14,24 \\
\hline Niacin (B3 / PP), mg & 31 & 45,5 & 40 \\
\hline Choline (B4), mg & 870 & 940 & 620 \\
\hline Pantothenic acid (B5), mg & 24,64 & 55 & 30 \\
\hline B6, mg & 2,74 & 4 & 4,54 \\
\hline Biotin (B7), mg & 0,1 & 0,12 & 0,15 \\
\hline Inositol (B8), mg & 290 & 465 & 300 \\
\hline Folic acid (B9), mg & 0,93 & 0,74 & 0,83 \\
\hline B12, mg & 0,01 & 0,02 & 0,02 \\
\hline Other components, g & 11,55 & 6,36 & 9,2 \\
\hline
\end{tabular}

The composition presented in the table contains the main components per $1 \mathrm{~kg}$ of the finished product. Other ingredients mean a complex of fat-soluble vitamins (A, D, E, K), minerals (Magnesium, Calcium, Zinc, etc.), fatty acids (linoleic, arachidonic, etc.), as well as prebiotics.

\section{Results and discussion}

Mixing of the composition according to the first recipe was carried out alternately on the base mixer and the developed one, varying their design and operating parameters. The range of operating parameters is shown in Table 2.

Table 2. Operating parameters of mixers.

\begin{tabular}{|c|c|c|c|}
\hline \multicolumn{2}{|c|}{ Constructive } & \multicolumn{2}{c|}{ Regime } \\
\hline $\begin{array}{c}\text { Taper angle } \\
(\boldsymbol{\alpha})\end{array}$ & Length of generatrix (h), cm & Rotor speed (n), $\mathbf{m i n}^{-\mathbf{1}}$ & Load factor (K3), \% \\
\hline $110^{\circ}$ & 5 & 700 & 30 \\
\hline $115^{\circ}$ & 6 & 900 & 45 \\
\hline $120^{\circ}$ & 7 & 1100 & 60 \\
\hline
\end{tabular}

The taper angle, the length of the generatrix, the rotor speed, the load factor can affect the change in the concentration of the mixture components, and, consequently, the coefficient of inhomogeneity. Based on the data obtained, it is possible to consider the operating conditions of the mixers under which the lowest coefficient of heterogeneity is observed. They are shown in Table 3. 
Table 3. Rational parameters of the mixers.

\begin{tabular}{|c|c|c|c|c|c|}
\hline \multirow{2}{*}{ Mixer design } & \multirow{2}{*}{$\mathbf{V}_{\mathbf{c}}, \mathbf{\%}$} & \multicolumn{4}{|c|}{ Parameters } \\
\cline { 3 - 6 } & & $\boldsymbol{\alpha}$ & $\mathbf{h}, \mathbf{c m}$ & $\mathbf{n}, \mathbf{m i n}^{-1}$ & $\mathbf{K}_{\mathbf{3}}, \mathbf{\%}$ \\
\hline Basic & 9,63 & $120^{\circ}$ & 6 & 900 & 45 \\
\hline Developed & 12,81 & $120^{\circ}$ & 6 & 900 & 45 \\
\hline
\end{tabular}

The lowest coefficient of heterogeneity of the basic mixer was $12,81 \%$ and the developed one was $9,63 \%$, with a difference of about three percent. From the obtained experimental data, it can be concluded that the rational operating parameters for both mixers are the same. Therefore, further mixing was carried out at these values, namely: the taper angle was $120^{\circ}$, the length of the generatrix was $6 \mathrm{~cm}$, the rotor speed was $900 \mathrm{~min}$ 1 , the load factor was $45 \%$. The coefficient of heterogeneity of the base mixer for the second and third recipes was $13,22 \%$ and $12,73 \%$, respectively. For the developed mixer according to the second and third recipes $-9,85 \%$ and $9,72 \%$, respectively. It should be noted that in all cases, in the first 6-8 samples, the key component is missing. This is due to the fact that the key component after the start of operation of the equipment takes time to pass through the mixer and distribute in the material flow. Figure 3 below shows the graphs of the dependence of the non-uniformity coefficient on the rotor speed when the load factor changes. As can be seen from the graphs, when the mixer is loaded at $45 \%$, the rotor speed has practically no effect on the coefficient of heterogeneity. At $60 \%$ mixer loading, a significant deterioration of the mix is observed. This may be due to the fact that a larger volume of the mixture does not have time to mix.

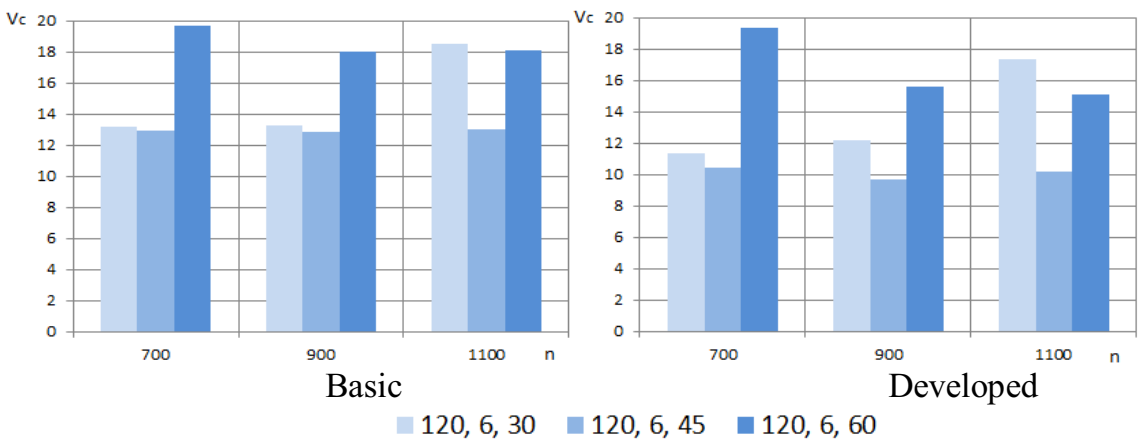

Fig. 3. Graph of the dependence of the coefficient of variation on the rotor speed when changing the load factor.

At a load of $30 \%$ and a rotor speed of 1100 min-1, a jump in the coefficient of variation occurs, since the mixture quickly reaches readiness, and the back mixing process begins, which contributes to a decrease in its quality. Figure 4 shows the graphs of the dependence of the coefficient of variation on the length of the generatrix, with a change in the taper angle. It can be seen in both graphs that the different length of the generatrix practically does not affect the coefficient of inhomogeneity. This may be due to the small range of length variation. But under equal conditions, the indicators of the coefficient of variation for the base and developed mixer significantly differ. The latter's indicators are 2-3\% less. 


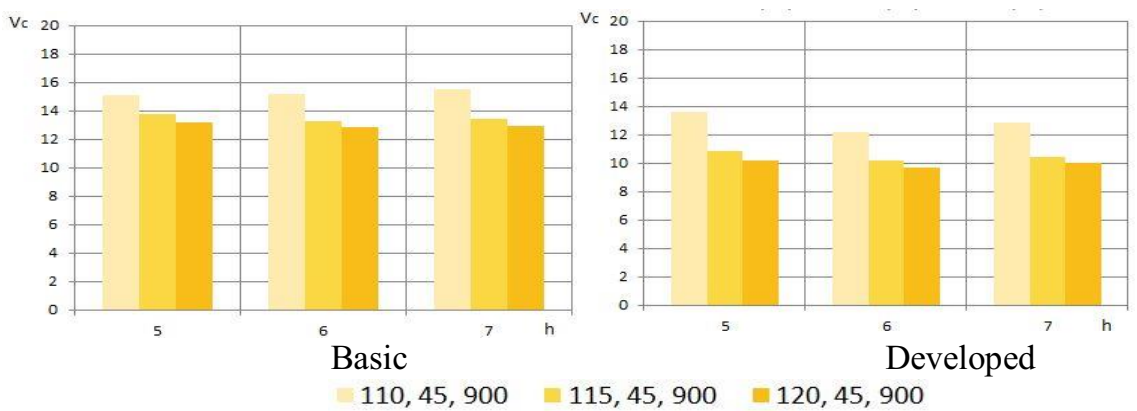

Fig. 4. Graph of the dependence of the coefficient of variation on the length of the generatrix when changing the taper angle.

In all three studies, the coefficient of heterogeneity of the base mixer was higher by an average of $3 \%$. All three indices of the coefficient of heterogeneity for the developed mixer do not exceed $10 \%$. This suggests that the mixture obtained on it can be characterized as a mixture of satisfactory quality.

\section{Conclusions}

The quality of dry infant formula is of great importance, since more and more people, for one reason or another, resort to using them. Today on the market there are a huge number of different names intended for children of different ages and physiological characteristics. Each manufacturer is trying to improve its recipe by adding various vitamins and additives to the mixture. At this stage, the question arises about the uniform distribution of a small amount of a component in a large volume of the mixture. We have carried out research on two mixers of different designs: basic and developed. With the help of each mixer, three different mixtures were obtained with different composition of components, including the key substance. The latter was taurine. To determine the composition of the resulting mixtures of taurine, 60 samples were taken. Each sample was quantitatively analyzed for the presence of a key component using HPLC. And then the coefficient of heterogeneity is calculated for each mixer. A comparative analysis of the work of the mixers showed that the rational parameters of operation for both devices will be: taper angle $-120^{\circ}$, generatrix length $-6 \mathrm{~cm}$, rotor speed - $900 \mathrm{~min}-1$, load factor $-45 \%$. For the first, second and third formulations of the mixture, the coefficient of heterogeneity of the base mixer was $12,81 \%$, $13,22 \%$ and $12,73 \%$, respectively. The coefficient of heterogeneity of the developed mixer was $9,63 \%$ for the first recipe, $9,85 \%$ and $9,72 \%$ for the second and third, respectively. The proposed mixer design variant showed better coefficients of heterogeneity than its prototype. The difference is about three percent, which is significant in the production of such a product as powdered infant formula.

\section{References}

1. E. Garvey, Critical Reviews in Food Science and Nutrition, 60 (7), 1160-1188 (2020), DOI: $10.1080 / 10408398.2018 .1562419$

2. C. Birch, International Journal of Food Science \& Technology, 54 (5), 1467-1485 (2019), DOI: $10.1111 /$ ijfs. 14060

3. Md. Momin, Foods and Raw Materials, 8(2), 422-428 (2020), DOI: 10.21603/23084057-2020-2-422-428

4. V. Ivanets, Foods and Raw Materials, 3(1), $62-69$ (2015), DOI: 10.12737/11239 
5. T.Varzakas, Critical Reviews in Food Science and Nutrition, 56(15), 2495-2510 (2016), DOI: 10.1080/10408398.2013.866070

6. D. Borodulin, Advances in Engineering Research, 685-689 (2018), DOI: 10.2991/agrosmart-18.2018.128

7. A. P. Burmistenkov, T. Ya. Belaya, and V. V. Korzun, 26 Continuous centrifugal mixer, Bul., 14 (USSR, 1985)

8. V. Bukhovets, Technique and Technology of Food Production, 49 (2), 193-200 (2019), DOI: 10.21603 / 2074-9414-2019-2-193-200

9. O. Pozdnyakova, Technique and Technology of Food Production, 48 (3), 90-95 (2018), DOI: 10.21603 / 2074-9414-2018-3-90-95

10. S. Misteneva, Food Industry, 8, 66-71 (2019), DOI: 10.24411 / 0235-2486-2019-10129

11. I. Dudarev, Lecture Notes in Mechanical Engineering, 54-64 (2020), DOI: 10.1007/978-3-030-22365-6_6

12. A. Kapranova, Studies in Systems, Decision and Control, 259, 81-91 (2020), DOI: 10.1007/978-3-030-32579-4_7

13. S. Cherpitskii, Chemical and Petroleum Engineering, 56 (1-2), 3-10 (2020), DOI: 10.1007/s10556-020-00731-w

14. A. Kapranova A., Lecture Notes in Civil Engineering, 70, 337-347 (2020), DOI: 10.1007/978-3-030-42351-3_30

15. E. Nevskaya, Foods and Raw Materials, 6 (1), 99-109 (2018), DOI: 10.21603/23084057-2018-1-99-109 